\section{Prostatakarzinom: Wer profitiert von GnRH-Antagonisten?}

- Ob und bei welchen Patienten die GnRH-Blocker die bislang mit Erfolg verwendeten $\mathrm{GnRH}$-Agonisten ersetzen werden, ist bislang unklar. Pharmakokinetische Vorteile von GnRH-Blockern wie Abarelix (Plenaxis ${ }^{\circledR}$ ) sind die rasche Testosteronsenkung ohne Flare-up-Phänomen, fehlende Microsurges (kurzfristige Testosteronerhöhungen) bei Folgeinjektionen sowie eine rasche Normalisierung des Testosteronspiegels nach Therapieende bzw. -unterbrechung. Für Abarelix wurden diese Vorteile im Vergleich zu GnRH-Agonisten sowohl als Monotherapie als auch in Kombination mit Antiandrogenen bestätigt.

Eine Indikation für die GnRH-Blocker könnte bei Patienten bestehen, bei denen unter dem kurzfristigen Testosteronanstieg durch GnRH-Agonisten die Beschwerden zunehmen. Momentan fehlen jedoch Untersuchungen, die zeigen, dass die genannten Charakteristika tatsächlich Vorteile für die
Patienten bringen. Für entsprechende Daten könnten Anwendungsbeobachtungen eine Rolle spielen. Klinische Studien kämen beim Prostatakarzinom nämlich oft zu wenig aussagekräftigen Ergebnissen, da der Verlauf zu günstig ist, um das Endziel Mortalität zu erreichen, erläuterte Prof. Dr. Lothar Weißbach, Berlin. Die ablative adjuvante oder neoadjuvante Homontherapie ist gemäß der neuen S3-Leitlinie beim metastasierten Prostatakarzinom Therapie der Wahl. Beim lokal fortgeschrittenen Prostatakarzinom ist die Hormonblockade als Alternative oder Ergänzung zur Bestrahlung beziehungsweise Operation indiziert.

LC

Symposium „Ohne Praxis keine Wissenschaft - Prostatakarzinom-Versorgungsforschung am Beispiel von Abarelix", anlässlich des 61. Kongress der Deutschen Gesellschaft für Urologie (DGU), Dresden, 18. September 2009; Veranstalter: Specialty European Pharma

\title{
Nierenzellkarzinom: Everolimus neuer Standard nach TKI-Versagen
}

- Mit Everolimus steht seit August 2009 eine neue zielgerichtete Therapie beim fortgeschrittenen Nierenzellkarzinom zur Verfügung. Der oral verfügbare Inhibitor wirkt dual und richtet sich gegen mTOR, ein Schlüsselenzym, das Tumorzellwachstum, -teilung und -stoffwechsel sowie Tumor-Angiogenese reguliert. Zugelassen für die Behandlung von Patienten, deren Tumor nicht oder nicht mehr auf Tyrosinkinase-Inhibitoren (TKI) anspricht, ist Everolimus (Afinitor $^{\circledR}$ ) der erste Wirkstoff mit erwiesener, in Phase III prospektiv geprüfter Wirksamkeit nach TKI-Versagen. In dieser Behandlungssituation wird Everolimus in den internationalen Therapieleitlinien als neuer Standard mit höchstem Evidenzlevel empfohlen, berichtete Prof. Dr. Jan Roigas, Berlin.

In der multizentrischen, randomisierten, placebokontrollierten RECORD-1-Studie behandelten Motzer und Mitarbeiter [Lancet 2008; 372: 449-56; Genitourinary Cancers Symposium, Orlando 2009; Abstract \# 278] 416 Patienten mit fortgeschrittenem Nierenzell- krebs nach Therapieversagen der Tyrosinkinase-Blockade mit diesem oral verfügbaren mTOR-Inhibitor (10 mg einmal täglich) oder Placebo. Das Ergebnis: Everolimus verlängerte signifikant das mediane progressionsfreie Überleben von 1,9 auf 4,9 Monate ( $p<0,001$; HR 0,33; $95 \%-K I$ 0,25-0,43). Nach zehn Monaten Behandlung zeigten mehr als ein Viertel der Patienten keine Krankheitsprogression. „In dieser erheblich vorbehandelten Patientengruppe ein sehr gutes Ergebnis“, kommentierte Roigas. Mit 2\% partieller Remission und $67 \%$ stabiler Erkrankung erreichte die objektive Ansprechrate $69 \%$. Und dies bei vergleichsweise guter Verträglichkeit. Rogas bezeichnete die Therapie als „gut durchführbare Therapie mit wenig Grad3/4-Nebenwirkungen“.

koc

Satelliten-Symposium „Neue Therapieoptionen beim Nierenzell- und Prostatakarzinom", anlässlich des 61. Kongress der Deutschen Gesellschaft für Urologie (DGU), Dresden, 18. September 2009, Veranstalter: Novartis Pharma
Geräte und Instrumente mild und gründlich reinigen

Ein mildalkalisches Flüssigreinigungmittel für die Instrumentenaufbereitung hat die BODE Chemie $\mathrm{GmbH}$ entwickelt. Dismoclean ${ }^{\circledR} 21$ clean ist gut materialverträglich und geht auch mit empfindlichen Oberflächen wie Aluminum, Messing und Kupfer schonend um. Außerdem kommt es völlig ohne zusätzliche Neutralisation aus. Es ist für Standmaschinen wie für Taktbandanlagen bei Temperaturen von $55^{-9}-90^{\circ} \mathrm{C}$ geeignet und entfernt sämtliche Anschmutzungen bei MIC- und chirurgischem Instrumentarium.

Nach Informationen von BODE Chemie

\section{Neue Website rund um die überaktive Blase}

Fast jeder fünfte Deutsche entwickelt in seinem Leben eine überaktive Blase. Obwohl die Symptome quälend sein und die Lebensqualität deutlich beeinträchtigen können, wird das Thema nach wie vor von Patienten wie Ärzten viel zu oft tabuisiert und totgeschwiegen. Die neue Website www.vesikur.de will Mut machen, das Problem offensiv anzugehen. Sie informiert umfassend über alle Aspekte der Dranginkontinenz - weit über das reine Lehrbuchwissen hinaus. Auch InfoBroschüren und Miktionstagebücher können hier online bestellt werden.

Nach Informationen von

Astellas Pharma

\section{Wartezimmerbroschüre für Patienten mit Prostatakarzinom}

„Prostatakrebs - was kommt nach der Hormontherapie?" lautet der Titel einer neuen Info-Broschüre, die sich an Patienten mit hormonrefraktärem Prostatakarzinom und ihre Angehörigen richtet. Sie will ihnen eine praktische Hilfestellung zum Umgang mit der Ekrankung bieten und kann über das Onkologie-Portal www.onkolleg.de kostenlos online bestellt werden.

Nach Informationen von Sanofi Aventis 